PROCEEDINGS OF THE

AMERICAN MATHEMATICAL SOCIETY

Volume 129, Number 4, Pages 1051-1056

S 0002-9939(00)05683-5

Article electronically published on October 10, 2000

\title{
NON-LINEARITY OF THE PLURICOMPLEX GREEN FUNCTION
}

\author{
FRANK WIKSTRÖM
}

(Communicated by Steven R. Bell)

\begin{abstract}
We consider the pluricomplex Green function with multiple poles as introduced by Lelong. We give a partial solution to a question concerning the set where the multipole Green function coincides with the sum of the corresponding single pole Green functions.
\end{abstract}

\section{INTRODUCTION}

The pluricomplex Green function with multiple poles was introduced by Lelong [8] in 1989, but has received relatively little attention until recently. For quite some time, no explicit example of a pluricomplex Green function with more than one pole was known. The first (partial) example is due to Carlehed [1] who computed the Green function for the bidisc with two poles, under the assumption that both poles are located on the plane $z_{2}=0$. At the time of writing, the Green function for the bidisc with two poles in general position is not known. In 1997 the first complete example, the two pole Green function for the ball, was computed independently by Coman [2] and Edigarian and Zwonek [5].

In this paper we give a partial solution to a problem by Lelong concerning the set where the multipole Green function is equal to the sum of the corresponding single pole functions. We give a complete characterization of this set for domains where the Green functions involved can be constructed using infima over analytic discs in the spirit of Lempert, i.e. for domains where the pluricomplex Green function coincides with the Lempert function $\delta$. There is a conjecture that this is the case for convex domains.

To start us off, let us recall the definition of the pluricomplex Green function.

Definition 1.1. Let $\Omega$ be a bounded domain in $\mathbb{C}^{n}$ and let $A=\left\{\left(w_{j}, \nu_{j}\right)\right\}_{j=1}^{p}$ be a finite subset of $\Omega \times \mathbb{R}_{+}$. Let $\mathcal{L}_{A}$ denote the set of all plurisubharmonic functions on $\Omega$, having a logarithmic pole of weight (at least) $\nu_{j}$ at each $w_{j}$, i.e. for $u$ to be a member of $\mathcal{L}_{A}$, we require that

$$
u(\zeta)-\nu_{j} \log \left|\zeta-w_{j}\right| \leq O(1), \text { as } \zeta \rightarrow w_{j} .
$$

We define the pluricomplex Green function with poles in $A$ by

$$
g(z, A)=\sup \left\{u(z): u \leq 0, u \in \mathcal{L}_{A}\right\} .
$$

Received by the editors June 25, 1999.

2000 Mathematics Subject Classification. Primary 32U35; Secondary 32F45.

Key words and phrases. Pluricomplex Green function with multiple poles, analytic discs, invariant distances. 
The case when the pole set $A$ is a singleton has been studied extensively; see, for example, [3, 6]. It is a remarkable fact that the single pole Green function in convex domains can be constructed using one-variable techniques. More precisely, let $\Omega$ be a bounded domain (not necessarily convex, or even hyperconvex). Fix $w \in \Omega$ and consider the pluricomplex Green function $g(\cdot)=g(\cdot, w)$ with pole at $w$. If $\phi: \Delta \rightarrow \Omega$ is an analytic disc in $\Omega$, with $\phi(0)=w$, then $g \circ \phi$ is a subharmonic function on the unit disc in $\mathbb{C}$. Obviously, $g \circ \phi$ is negative and has a logarithmic pole at 0 . Hence, $g \circ \phi$ is a member in the defining family for the Green function on $\Delta$ with pole at 0 , and thus $g(\phi(\zeta)) \leq \log |\zeta|$. Let us introduce the following notation:

Definition 1.2. Let $\Omega$ be a bounded domain in $\mathbb{C}^{n}$. Define the Lempert function $\delta: \Omega \times \Omega \rightarrow \mathbb{R} \cup\{-\infty\}$ by

$$
\delta(z, w)=\inf \{\log |\zeta|: \exists f \in \mathcal{O}(\Delta, \Omega) \text {, s.t. } f(0)=z, f(\zeta)=w\},
$$

where $\mathcal{O}(\Delta, \Omega)$ denotes the set of all holomorphic mappings from the unit disc $\Delta \subset \mathbb{C}$ into $\Omega$.

Clearly, from the above discussion, we always have that $g \leq \delta$. It is a deep theorem due to Lempert [9], that for convex domains, $g=\delta$. Therefore, it is tempting to try a similar approach to deal with the multipole Green function and it is natural to extend the definition of $\delta$ to cover this case.

Definition 1.3. Let $\Omega$ be a bounded domain in $\mathbb{C}^{n}$ and let $A=\left\{\left(w_{j}, \nu_{j}\right)\right\}_{j=1}^{p}$ be a finite subset of $\Omega \times \mathbb{R}_{+}$. Define

$$
\delta(z, A)=\inf \left\{\sum_{j=1}^{p} \nu_{j} \log \left|\zeta_{j}\right|: \exists f \in \mathcal{O}(\Delta, \Omega) \text {, s.t. } f(0)=z, f\left(\zeta_{j}\right)=w_{j}, \forall j\right\} .
$$

In some situations, it is more convenient to instead study $\delta^{A}$, where

$$
\delta^{A}(z)=\min _{\emptyset \neq B \subseteq A} \delta(z, B),
$$

but we will see later on (cf. Theorem 2.4) that for convex domains, $\delta(z, A)=\delta^{A}(z)$.

Note that if $\Omega$ is a bounded domain and $A$ is a finite subset of $\Omega \times \mathbb{R}_{+}$, then $\delta(z, A)<0$, since it is always possible to pass an analytic disc through $z$ and $A$. As in the case of one pole, it is straightforward to verify that $g(z, A) \leq \delta(z, A)$. Coman 2 has shown that if $\Omega$ is a ball in $\mathbb{C}^{n}$, and $A$ consists of two points of equal weight, then $g(z, A)=\delta(z, A)$. It seems reasonable to conjecture that this equality holds in every convex domain.

Conjecture 1.4. Let $\Omega$ be a bounded convex domain in $\mathbb{C}^{n}$, and let $A$ be a finite subset of $\Omega \times \mathbb{R}_{+}$. Then $g(z, A)=\delta(z, A)$.

Recently, there have been other similar approaches to understanding the Green function with multiple poles better using "disc formulas"; see Edigarian 4] and Lárusson and Sigurdsson [7].

\section{Some Properties of the Lempert FunCtion}

The most natural setting to study the Lempert function is in taut domains. Every hyperconvex domain (recall that a domain is hyperconvex if it admits a bounded plurisubharmonic exhaustion function) is taut, so in connection with the 
pluricomplex Green function, we do not lose very much by restricting ourselves to this class of domains. We recall the definition of tautness.

Definition 2.1. Let $\Omega$ be a domain in $\mathbb{C}^{n}$. We say that $\Omega$ is taut if for any sequence $f_{n} \in \mathcal{O}(\Delta, \Omega)$ there exists a subsequence $f_{n_{j}}$ such that either

- $f_{n_{j}} \rightarrow f \in \mathcal{O}(\Delta, \Omega)$ locally uniformly, or

- for each compact subset $K$ of $\Delta$ and each compact subset $L$ of $\Omega, f_{n_{j}}(K) \cap L$ is empty for all sufficiently large $j$.

Recall that the Lempert function is defined as an infimum over a large family of discs. An interesting and important question is whether this infimum is attained by a specific disc or not. It is not difficult to prove, using a normal family argument, that in the case of one pole, the infimum is always attained. Moreover, Lempert proved that if $\Omega$ is strictly convex, it is attained by an essentially unique disc. In the case of several poles, we can prove the following:

Theorem 2.2. Let $\Omega$ be a bounded taut domain, and let $A$ be a finite subset of $\Omega \times \mathbb{R}_{+}$containing at least two points. If $\delta(z, A)$ is not attained by any analytic disc, then

$$
\delta(z, A) \geq \min _{A^{\prime} \subsetneq A} \delta\left(z, A^{\prime}\right) .
$$

Proof. Let $A=\left\{\left(w_{j}, \nu_{j}\right)\right\}_{j=1}^{p}$. Take a sequence of analytic discs $\phi_{k}$, where $\phi_{k}(0)=$ $z$, and $\phi_{k}\left(\zeta_{j}^{(k)}\right)=w_{j}$ for every $k$ and every $1 \leq j \leq p$, where $\sum_{j} \nu_{j} \log \left|\zeta_{j}^{(k)}\right|$ converges to $\delta(z, A)$, as $k \rightarrow \infty$.

By passing to a subsequence, using that $\Omega$ is taut, we may assume that $\phi_{k}$ converges locally uniformly to some $\phi \in \mathcal{O}(\Delta, \Omega)$. Also (if necessary, by passing to a subsequence again), we may assume that $\zeta_{j}^{(k)} \rightarrow \zeta_{j} \in \bar{\Delta}$, for each $j$ as $k \rightarrow \infty$.

If no $\zeta_{j} \in \partial \Delta, \phi$ is an analytic disc attaining the infimum in the definition of $\delta(z, A)$. Otherwise, assume that, say, $\zeta_{j} \in \Delta$ for $1 \leq j \leq m$ and $\zeta_{j} \in \partial \Delta$ for $m+1 \leq$ $j \leq p$. (Note that not every $\zeta_{j}$ can be in $\partial \Delta$, as this would imply that $\delta(z, A)=0$.) Then $\phi$ is a member in the defining family for $\delta\left(z,\left\{\left(w_{1}, \nu_{1}\right), \ldots,\left(w_{m}, \nu_{m}\right)\right\}\right)$, and thus $\delta(z, A) \geq \delta\left(z,\left\{\left(w_{1}, \nu_{1}\right), \ldots,\left(w_{m}, \nu_{m}\right)\right\}\right)$.

Corollary 2.3. Let $\Omega$ be a bounded taut domain in $\mathbb{C}^{n}$, and let $A$ be a finite subset of $\Omega$. Then for every $z \in \Omega$ there exists an analytic disc $\phi$, such that $\phi(0)=z$, passing through a (non-empty) subset $\left\{w_{j_{1}}, \ldots, w_{j_{m}}\right\}$ of $\left\{w_{1}, \ldots, w_{p}\right\}$, such that $\phi$ attains the infimum in the definition of $\delta^{A}(z)=\delta\left(z,\left\{\left(w_{j_{1}}, \nu_{j_{1}}\right), \ldots,\left(w_{j_{m}}, \nu_{j_{m}}\right)\right\}\right)$.

Proof. If $A$ is a singleton, a normal family argument close to the one used in the previous proof will show that the corollary is true for this case.

Otherwise, by the previous theorem, either there is an analytic disc attaining the infimum, or $\delta^{A}(z, A)=\delta(z, B)$ for some proper subset $B \subset A$, and $\delta(z, B)$ is attained by an analytic disc passing through $z$ and the points in $B$.

We will use the term extremal disc (with respect to $z$ and $A$ ) for any analytic disc satisfying the conclusions of Corollary 2.3. If $A$ is a singleton and $\Omega$ is convex, these extremal discs are geodesics for the Kobayashi metric, and the pluricomplex Green function is harmonic along the extremal discs. We call these discs complex geodesics.

In the multipole case, it is not known whether the pluricomplex Green function is harmonic along the extremal discs, but using a maximum principle argument, it 
is not difficult to show that if $\delta(z, A)=g(z, A)$ for some $z \in \Omega$ ( $\Omega$ not necessarily being convex), then the equality holds along any extremal disc for $z$ and $A$, and the pluricomplex Green function is harmonic along this disc. Also, in this case the extremal disc will be extremal with respect to any point in its image and $A$.

Let us conclude this section by showing that, for bounded convex domains, $\delta(z, A)=\delta^{A}(z)$.

Theorem 2.4. Let $\Omega$ be a bounded convex domain in $\mathbb{C}^{n}$, and let $A=\left\{\left(w_{j}, \nu_{j}\right)\right\}_{j=1}^{p}$ be a finite subset of $\Omega \times \mathbb{R}_{+}$. Then $\delta(z, A)=\delta^{A}(z)$ for all $z \in \Omega$.

Proof. Clearly, $\delta^{A}(z) \leq \delta(z, A)$. Conversely, fix any $B \subset A$. For simplicity of notation, let us assume that $B=\left\{\left(w_{j}, \nu_{j}\right)\right\}_{j=1}^{m}, m<p$, and let $\phi$ be an analytic disc, such that $\phi(0)=z, \phi\left(\zeta_{j}\right)=w_{j}, 1 \leq j \leq m$.

Let $r>1$, where $r$ is so small that $r \zeta_{j} \in \Delta$ for every $j$, and define $\phi_{r}: \Delta \rightarrow \Omega$ by $\phi_{r}(\zeta)=\phi\left(r^{-1} \zeta\right)$. Define $K$ as the convex hull of $\phi_{r}(\bar{\Delta}) \cup\left\{w_{m+1}\right\}$. Since $\phi_{r}(\bar{\Delta}) \cup\left\{w_{m+1}\right\} \subset \subset \Omega$, we can find an $\varepsilon>0$, such that the distance between $K$ and $\partial \Omega$ is at least $2 d \varepsilon$, where $d=\max _{\zeta \in \bar{\Delta}}\left|w_{m+1}-\phi_{r}(\zeta)\right|$.

Define

$$
f(\zeta)=\phi_{r}(\zeta)+a(\zeta)\left(w_{m+1}-\phi_{r}(\zeta)\right),
$$

where $a$ is a holomorphic function on $\Delta$ satisfying

1. $-\varepsilon<\operatorname{Re} a<1+\varepsilon$,

2. $|\operatorname{Im} a|<\varepsilon$,

3. $a(0)=0$,

4. $a\left(r \zeta_{j}\right)=0$, for $1 \leq j \leq m$,

5. $a\left(\zeta^{*}\right)=1$, for some $\zeta^{*}$ with $\left|\zeta^{*}\right|>1 / r$.

Let us assume that such a function $a$ exists. Then $f(\zeta)=f_{1}(\zeta)+f_{2}(\zeta)$, where $f_{1}(\zeta)=\phi_{r}(\zeta)+(\operatorname{Re} a(\zeta))\left(w_{m+1}-\phi_{r}(\zeta)\right)$ and $f_{2}(\zeta)=i(\operatorname{Im} a(\zeta))\left(w_{m+1}-\phi_{r}(\zeta)\right)$. Now, $f_{1}(\zeta) \in K+B(0, d \varepsilon)$, and $f_{2}(\zeta) \in B(0, d \varepsilon)$. Hence $f(\zeta) \in K+B(0,2 d \varepsilon) \subset \Omega$, and $f(0)=z, f\left(r \zeta_{j}\right)=w_{j}, 1 \leq j \leq m, f\left(\zeta^{*}\right)=w_{m+1}$. Consequently,

$$
\delta\left(z, B \cup\left\{\left(w_{m+1}, \nu_{m+1}\right)\right\}\right) \leq \sum_{j=1}^{m} \nu_{j} \log \left|r \zeta_{j}\right|+\nu_{m+1} \log \left|\zeta^{*}\right| .
$$

Letting $r \rightarrow 1$, we see that

$$
\delta\left(z, B \cup\left\{\left(w_{m+1}, \nu_{m+1}\right)\right\}\right) \leq \sum_{j=1}^{m} \nu_{j} \log \left|\zeta_{j}\right|,
$$

and by taking the infimum over all possible discs $\phi$, it follows that

$$
\delta\left(z, B \cup\left\{\left(w_{m+1}, \nu_{m+1}\right)\right\}\right) \leq \delta(z, B) .
$$

Repeating this construction, adding one point at a time, we conclude that $\delta(z, A) \leq$ $\delta(z, B)$. To finish the proof, we only have to prove the existence of a holomorphic function $a$ with the properties above.

To do this, let $D$ be a bounded simply connected domain with real-analytic boundary in $\mathbb{C}$, such that $\{0,1\} \subset D \subset\{z:-\varepsilon<\operatorname{Re} z<1+\varepsilon,|\operatorname{Im} z|<\varepsilon\}$. Let $\rho_{D}$ be a Riemann mapping $\Delta \rightarrow D$, such that $\rho_{D}(0)=0$. Define $\gamma: \Delta \rightarrow \Delta$, by

$$
\gamma(\zeta)=\zeta \prod_{j=1}^{m} \frac{\zeta-r \zeta_{j}}{1-r \bar{\zeta}_{j} \zeta}
$$


Note that $\gamma$ is a proper mapping from $\Delta$ onto $\Delta$, and there is some $\zeta^{*} \in \Delta$, such that $\rho_{D}\left(\gamma\left(\zeta^{*}\right)\right)=1$. In the construction of $D$, by making sure that 1 is sufficiently close to $\partial D$, we can assume that $\left|\zeta^{*}\right|>1 / r$. Finally, letting $a=\rho_{D} \circ \gamma$, we obtain a holomorphic function $a: \Delta \rightarrow D$, such that $a(0)=\rho_{D}(0)=0, a\left(r \zeta_{j}\right)=\rho_{D}(0)=0$, and $a\left(\zeta^{*}\right)=1$.

\section{LELONG'S QUESTION}

How can we estimate the multipole Green function? If $A=\left\{\left(w_{j}, \nu_{j}\right)\right\}_{j=1}^{p} \subset$ $\Omega \times \mathbb{R}_{+}$, we have one immediate estimate, namely

$$
\sum_{j=1}^{p} \nu_{j} g\left(z, w_{j}\right) \leq g(z, A) \leq \min _{B \subseteq A} g(z, B) .
$$

This follows directly from the definition of the different Green functions. The function on the leftmost side is a member in the defining family for $g(\cdot, A)$ and similarly, $g(\cdot, A)$ is in the defining family for each of the functions in the rightmost side. With a similar reasoning, we note that

$$
\sum_{j=1}^{p} \nu_{j} \delta\left(z, w_{j}\right) \leq \delta(z, A)
$$

Lelong [8] considered the question when the first inequality in (3.1) is in fact an equality. He showed that the set $E_{g}=\left\{z: \sum_{j} \nu_{j} g\left(z, w_{j}\right)=g(z, A)\right\}$ is closed and has no isolated points. For the ball, he also showed that $E_{g}$ has empty interior and conjectured that this is always the case. Carlehed [1] pointed out that Lelong's conjecture fails for the bidisc and the case of two poles. Carlehed also noted that if the domain is strictly convex and $A$ contains two points, the set $E_{g}$ is never empty, since it must contain the complex geodesic connecting the two poles. From his arguments it follows that the set $E_{g}$ for the bidisc has non-empty interior since it must contain every extremal disc connecting the two points. The set $E_{g}$ can be thought of as a way of measuring the non-linearity of $g$ and/or the complex Monge-Ampère operator.

Let us generalize these results.

Theorem 3.1. Let $\Omega$ be a bounded convex domain in $\mathbb{C}^{n}$ and let $A=\left\{\left(w_{1}, \nu_{1}\right)\right.$, $\left.\left(w_{2}, \nu_{2}\right)\right\}$ be a subset of $\Omega \times \mathbb{R}_{+}$consisting of two points. The set $E_{\delta}=\{z \in$ $\left.\Omega: \delta(z, A)=\nu_{1} \delta\left(z, w_{1}\right)+\nu_{2} \delta\left(z, w_{2}\right)\right\}$ is equal to the union of (the images of) all complex geodesics connecting $w_{1}$ and $w_{2}$.

Proof. Let $\phi$ be a complex geodesic connecting $w_{1}$ and $w_{2}$. By the invariance result of Lempert, this disc is also extremal for any pair of points in $\phi(\Delta)$. Take $z \in \phi(\Delta)$. By a change of coordinates, we may assume that $z=\phi(0)$ and $\phi\left(\zeta_{j}\right)=w_{j}, j=1,2$. Since $\phi$ is extremal for $\left(z, w_{1}\right)$ and $\left(z, w_{2}\right)$, we have that $\log \left|\zeta_{j}\right|=\delta\left(z, w_{j}\right)$. On the other hand, from the definition of $\delta(z, A)$, we see that $\delta(z, A) \leq \nu_{1} \log \left|\zeta_{1}\right|+$ $\nu_{2} \log \left|\zeta_{2}\right|=\nu_{1} \delta\left(z, w_{1}\right)+\nu_{2} \delta\left(z, w_{2}\right)$. Using (3.2), we can conclude that $\delta(z, A)=$ $\nu_{1} \delta\left(z, w_{1}\right)+\nu_{2} \delta\left(z, w_{2}\right)$.

For the converse, assume that $z \in E_{\delta}$. Since $z \in E_{\delta}$, and $\delta\left(z, w_{j}\right)=g\left(z, w_{j}\right)<0$,

$$
\delta(z, A)<\min \left\{\nu_{1} \delta\left(z, w_{1}\right), \nu_{2} \delta\left(z, w_{2}\right)\right\} .
$$

This implies (Theorem [2.2) that there is an extremal disc, $\phi$, w.r.t. $z$ and $A$ passing through both $w_{1}$ and $w_{2}$. More precisely, $\phi(0)=z, \phi\left(\zeta_{j}\right)=w_{j}, j=1,2$, and $\nu_{1} \log \left|\zeta_{1}\right|+\nu_{2} \log \left|\zeta_{2}\right|=\delta(z, A)$ 
By assumption $\nu_{1} \delta\left(z, w_{1}\right)+\nu_{2} \delta\left(z, w_{2}\right)=\delta(z, A)=\nu_{1} \log \left|\zeta_{1}\right|+\nu_{2} \log \left|\zeta_{2}\right|$, but since $\phi$ is a member of the defining family for both $\delta\left(\cdot, w_{j}\right), \delta\left(z, w_{j}\right) \leq \log \left|\zeta_{j}\right|$, this last inequality must in fact be an equality. Hence, $\phi$ is a complex geodesic connecting $w_{1}$ and $w_{2}$.

Remark. The above proof is easily modified to the case of more than two poles. The corresponding condition is then that $E_{\delta}=\left\{z: \delta(z, A)=\sum_{w_{j} \in A} \nu_{j} \delta\left(z, w_{j}\right)\right\}$ equals the set of complex geodesics connecting $w_{1}$ and $w_{2}$ and passing through all the $w_{j}$ 's. Also, we see that, for convex domains, $E_{\delta} \subseteq E_{g}$. If $z \in E_{\delta}$, we have that

$$
g(z, A) \leq \delta(z, A)=\sum_{j=1}^{p} \nu_{j} \delta\left(z, w_{j}\right)=\sum_{j=1}^{p} \nu_{j} g\left(z, w_{j}\right) .
$$

To prove that $E_{\delta}=E_{g}$, at least with a similar method, we must assume that $\delta(z, A)=g(z, A)$, but if we believe in Conjecture 1.4 this is true for every convex domain. Anyway, for domains where $\delta=g$, Theorem 3.1 holds with $E_{\delta}$ replaced by $E_{g}$.

If we assume that $\Omega$ is strictly convex, there is a unique complex geodesic connecting $w_{1}$ and $w_{2}$, and in this case $E_{\delta}$ is a single analytic disc.

In the case when we have more than two poles, the set $E_{\delta}$ is generically empty (with a suitable interpretation of what is happening at the poles). The only possibility that $E_{\delta}$ is non-trivial when $A$ contains three points, is when all poles lie on a common complex geodesic.

It is also interesting to note that the set $E_{\delta}$ is independent of the weights $\nu_{j}$, at least in convex domains. Indeed, Theorem 3.1 shows that $E_{\delta}$ only depends on the location of the poles, not on their respective weights.

\section{REFERENCES}

1. M. Carlehed, Some properties of the pluricomplex Green function and potentials, Research reports No 14, 1995. Dept. of Mathematics, Umeå University.

2. D. Coman, The pluricomplex Green function with two poles of the unit ball of $\mathbb{C}^{n}$, Pacific J. Math. (to appear).

3. J. P. Demailly, Mesures de Monge-Ampère et mesures pluriharmoniques, Math. Z. 194 (1987), 519-564. MR 88g:32034

4. A. Edigarian, On definitions of the pluricomplex Green function, Ann. Polon. Math. 67 (1997), no. 3, 233-246. MR 99f:32039

5. A. Edigarian and W. Zwonek, Invariance of the pluricomplex Green function under proper mappings with applications, Complex Variables Theory Appl. 35 (1998), 367-380. MR 99d:32017

6. M. Klimek, Extremal plurisubharmonic functions and invariant pseudodistances, Bull. Soc. Math. France 113 (1985), 231-240. MR 87d:32032

7. F. Lárusson and R. Sigurdsson, Plurisubharmonic functions and analytic discs on manifolds, J. Reine Angew. Math. 501 (1998), 1-39. MR 99e:32020

8. P. Lelong, Fonction de Green pluricomplexe et lemme de Schwarz dans les espaces de Banach, J. Math. Pures Appl. 68 (1989), 319-347. MR 91c:46065

9. L. Lempert, La métrique de Kobayashi et la représentation des domaines sur la boule, Bull. Soc. Math. France 109 (1981), 427-474. MR 84d:32036

Department of Mathematics, Ume̊ University, S-901 87 Ume̊̊, Sweden

E-mail address: Frank.Wikstrom@math.umu.se 